\title{
Genome-wide annotation and analysis of zebra finch microRNA repertoire reveal sex-biased expression
}

\author{
Guan-Zheng Luo ${ }^{1 \dagger}$, Markus Hafner ${ }^{2 \dagger}$, Zhimin Shi ${ }^{3}$, Miguel Brown ${ }^{2}$, Gui-Hai Feng ${ }^{1}$, Thomas Tuschl2,
} Xiu-Jie Wang ${ }^{1 *}$ and XiaoChing $\mathrm{Li}^{3^{*}}$

\begin{abstract}
Background: MicroRNAs (miRNAs) are small noncoding RNAs that regulate gene expression post-transcriptionally in a wide range of biological processes. The zebra finch (Taeniopygia guttata), an oscine songbird with characteristic learned vocal behavior, provides biologists a unique model system for studying vocal behavior, sexually dimorphic brain development and functions, and comparative genomics.

Results: We deep sequenced small RNA libraries made from the brain, heart, liver, and muscle tissues of adult male and female zebra finches. By mapping the sequence reads to the zebra finch genome and to known miRNAs in miRBase, we annotated a total of 193 miRNAs. Among them, 29 (15\%) are avian specific, including three novel zebra finch specific miRNAs. Many of the miRNAs exhibit sequence heterogeneity including length variations, untemplated terminal nucleotide additions, and internal substitution events occurring at the uridine nucleotide within a GGU motif. We also identified seven Z chromosome-encoded miRNAs. Among them, miR-2954, an avian specific miRNA, is expressed at significantly higher levels in males than in females in all tissues examined. Target prediction analysis reveals that miR-2954, but not other Z-linked miRNAs, preferentially targets Z chromosome-encoded genes, including several genes known to be expressed in a sexually dimorphic manner in the zebra finch brain.

Conclusions: Our genome-wide systematic analysis of mature sequences, genomic locations, evolutionary sequence conservation, and tissue expression profiles of the zebra finch miRNA repertoire provides a valuable resource to the research community. Our analysis also reveals a miRNA-mediated mechanism that potentially regulates sex-biased gene expression in avian species.
\end{abstract}

Keywords: Zebra finch, miRNAs, Sequence variations, Tissue-enriched miRNA expression, Z chromosome, Sex-biased miRNA expression

\section{Background}

MicroRNAs (miRNAs) are short non-coding RNA molecules that regulate gene expression post-transcriptionally [1]. miRNAs are transcribed by RNA polymerase II. Primary transcripts of miRNAs are cleaved in the nucleus by the nuclease Drosha to generate precursor miRNAs (premiRNAs) with a characteristic hairpin-like secondary

\footnotetext{
* Correspondence: xjwang@genetics.ac.cn; Xli4@|suhsc.edu

${ }^{\dagger}$ Equal contributors

'State Kay Laboratory of Plant Genomics, Institute of Genetics and Developmental Biology, Chinese Academy of Sciences, Beijing 100101, China ${ }^{3}$ Neuroscience Center of Excellence, Louisiana State University Health Sciences Center, New Orleans, LA 70112, USA

Full list of author information is available at the end of the article
}

structure [2-4]. The pre-miRNAs are then exported to the cytoplasm and further cleaved by the RNase III enzyme Dicer to release a 21-23 nt small RNA duplex from the stem region of the hairpins [2,5-7]. In most cases, only one strand of the small RNA duplex is retained as mature miRNA, and the other strand, termed miRNA star (miRNA*), is degraded [8]. In some cases, however, both strands from the same hairpin are retained as functional miRNAs, which are annotated as miRNA-5p and miRNA-3p, respectively, depending on which arm of the hairpin they are derived [9]. Sequence variations, such as nucleotide deletion, insertion, untemplated terminal extension, and editing, have been observed

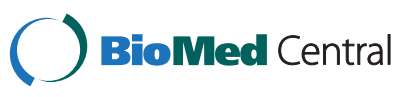


for some miRNAs [10-13]. The majority of animal miRNAs function by binding to the 3 -untranslated regions (UTRs) of target mRNAs via sequence complementarity to induce mRNA degradation or repress protein translation [14-16]. miRNAs have been found in most eukaryotes and many of them are conserved through evolution. The recent application of deep sequencing technology has also revealed an increasing number of species-specific miRNAs [10,17-21]. It is estimated that over $60 \%$ of human protein-coding genes might be regulated by miRNAs [22]. By finetuning gene expression, miRNAs regulate many biological processes ranging from cell proliferation, cell fate specification, cell differentiation, apoptosis, animal development, metabolism, to various disease conditions $[1,16,23]$.

The zebra finch (Taeniopygia guttata), an oscine songbird, provides a unique animal model for neurobiological research with its characteristic learned vocal behavior [24]. Zebra finches use a discrete set of interconnected brain nuclei and pathways, commonly referred to as the song system, to control song behavior [25]. Song behavior in zebra finches is sexually dimorphic: only male birds sing, and the song system is highly developed in males [26,27]. For decades, zebra finches have been widely used to study vocal learning, neuronal replacement, and sexually dimorphic development of male and female brains $[24,28]$. In recent years, large efforts have been devoted to developing genomic resources and extending research in zebra finches to molecular and genomics levels [29-35]. As part of this effort, we have systematically characterized miRNA expression in various tissues of adult female and male zebra finches using the Illumina high throughput sequencing platform. Taking advantage of the large sequence dataset now available, we analyzed features of these miRNAs, including miRNA sequence conservation through evolution, miRNA clusters, tissue-enriched expression, and sequence variations. Previously, using the 454 sequencing platform, we detected a $\mathrm{Z}$ chromosome encoded miRNA, miR2954, in the male zebra finch brain [32]. This miRNA has recently been shown to be expressed in the auditory forebrain of zebra finches, and its expression is regulated by song exposure [35]. We have further explored the expression of miR-2954 and other $\mathrm{Z}$ chromosome encoded miRNAs in male and female tissues and have found that miR-2954 is predominantly expressed in all male tissues examined. Our target prediction analysis revealed that miR-2954, but not other Z chromosome encoded miRNAs, preferentially targets $\mathrm{Z}$ chromosome encoded genes.

\section{Results}

\section{General features of zebra finch miRNAs}

We prepared eight small RNA libraries from four tissues brain, heart, liver, and muscle - of adult female and male zebra finches. These libraries were sequenced using the Illumina Genome Analyzer II high throughput sequencing platform. We obtained a total of 23,366,676 raw sequence reads from all libraries combined. After adaptor trimming and removal of low quality reads and orphan sequences (single reads), 19,424,182 high quality sequence reads were retained for subsequent analysis (Additional file 1). Of these reads, $60 \%$ mapped perfectly to the zebra finch genome assembly (release 1.0). We extracted flanking sequences around mapped reads and used mFold [36] to search for hairpin-like secondary structures. A total of 169 mature miRNAs were identified, which exhibited good hairpin-like precursor structures and matched known miRNAs recorded in miRBase (version 17.0) with high sequence homology (identical or with one mismatch). Considering that the current zebra finch genome assembly is relatively new and may contain gaps [32], we compared the unmapped sequence reads to known miRNA sequences in miRBase, and identified 21 additional miRNAs with high sequence homology (identical or with one mismatch) to known miRNAs in other species. Following the general criteria for miRNA annotation [37], we identified three novel zebra finch specific miRNA candidates, which are supported by good hairpin-like precursor structures, presence of corresponding star sequences, and relatively high expression (Figure 1A). Taken together, we identified 193 distinct zebra finch miRNAs. The sequences, genomic locations, and relative expression levels of these miRNAs are summarized in Additional file 2.

miRNAs and their variants accounted for $\sim 64 \%$ of all high quality reads. The remaining sequence reads were classified as rRNA/tRNA fragments, repeatassociated small RNAs, degradation products of mRNAs, and undefined small RNAs (Figure 1B). The expression levels of individual miRNAs spanned a wide range, from a few copies to thousands of copies (Additional file 2). For example, tgu-miR-1, which was highly expressed in both heart and muscle, was represented by 2,541,528 reads, accounting for $25 \%$ of the total miRNA reads (Figure 1C), whereas the three novel zebra finch miRNAs were expressed at relatively low levels, represented by 304,66 , and 73 reads respectively (Figure 1A). (Note, these numbers represent combined totals from four tissues and two sexes. For read counts in individual tissues and sexes, see Additional file 2). All of the top 20 most abundantly expressed miRNAs were conserved in vertebrates, and they comprised $76 \%$ of the total miRNA sequence reads (Figure 1C).

\section{Potential avian specific miRNAs}

By comparing to mature miRNA sequences in miRBase, 33 of the 193 zebra finch miRNAs did not have homologs (identical or with one mismatch) outside of avian 


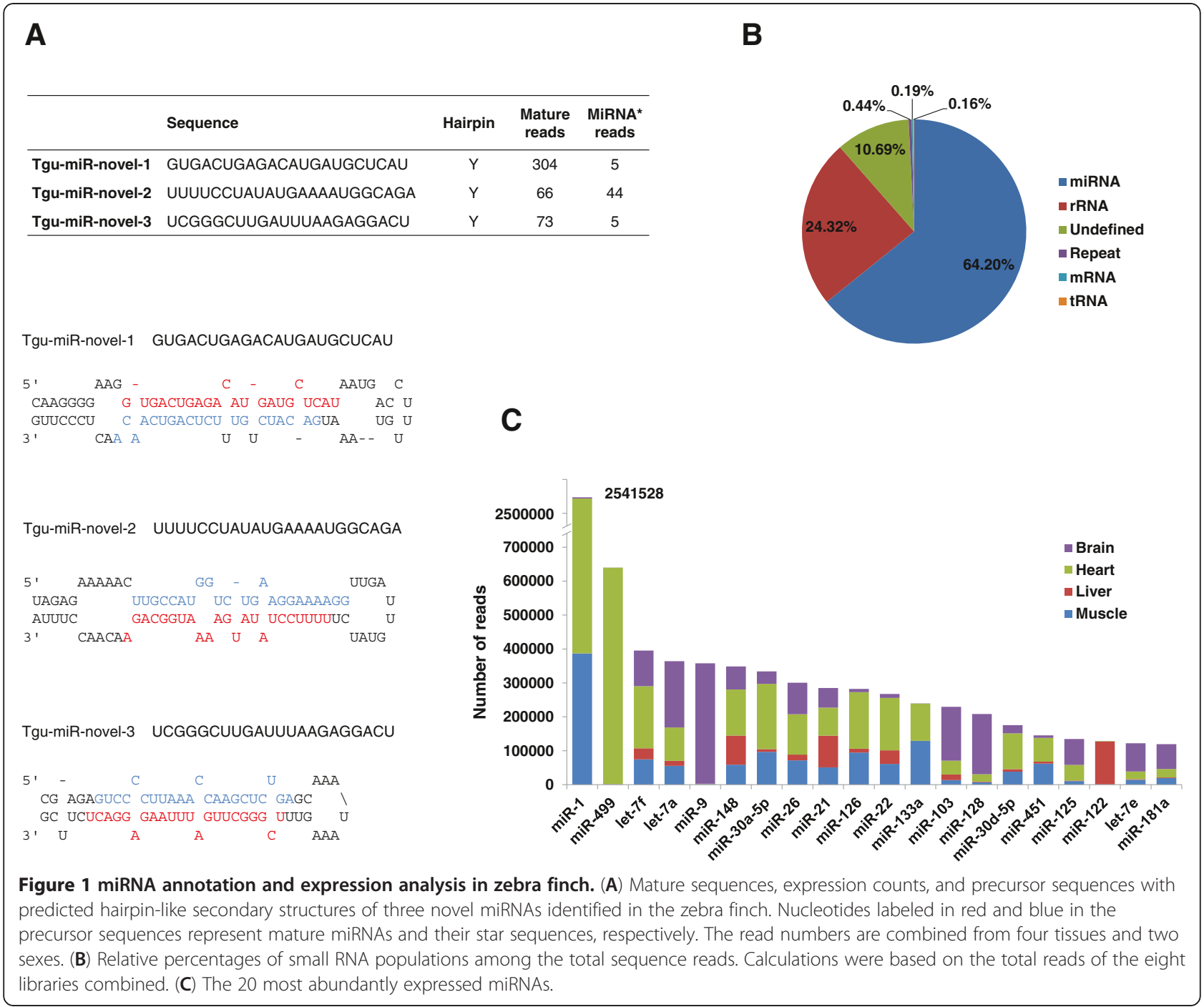

species. To investigate whether these 33 miRNAs had unidentified homologs in other genomes, we searched for homologous sequences in the genomes of 9 animal species including $C$. elegans, drosophila (D. melanogaster), zebrafish (Danio rerio), X. tropicalis (Xenopus tropicalis), lizard (Anolis carolinensis), platypus (Ornithorhynchus anatinus), chicken (Gallus gallus), mouse (Mus musculus), and human (Homo sapiens). Sequence homologs of 4 miRNAs (miR-2978, miR-2983, miR-2984, and miR-2987) were found in at least one non-avian vertebrate, and these were subsequently excluded from the list of avianspecific miRNAs. Thus, 29 miRNAs were classified as avian-specific miRNAs, of which 19 were zebra finch specific (Figure 2). By extending this analysis to all the 193 zebra finch miRNAs, we found that 37 miRNAs were conserved from $C$. elegans through humans, 103 were conserved in vertebrates, and 24 were only conserved between avian and mammals (Additional file 3).

\section{miRNA clusters}

Using a distance of $10 \mathrm{~kb}$ between any two miRNA genes as a cutoff, 87 miRNA genes were grouped into 36 genomic clusters. We named these miRNA gene clusters according to the name of the first member of each cluster followed by the number of miRNA genes within the cluster in parenthesis, e.g., cluster tgu-mir-24 (3) contains tgu-mir-24, tgu-mir-27b, and tgu-mir-23b (Additional file 4). Among these clusters, 3 appeared to be formed by tandem duplications of a single miRNA gene, e.g., the tgu-mir-2989(2) cluster on chromosome 8 contains 2 copies of the tgu-mir-2989 gene (Additional file 4). A majority of clusters were conserved in vertebrates (having more than 2 miRNA genes in the same cluster in other species); one cluster (tgu-let-7a-2(3)) was also conserved in D. melanogaster, but none was conserved in C. elegans. One cluster (tgu-mir-2989(2)) appeared to be zebra finch specific. 


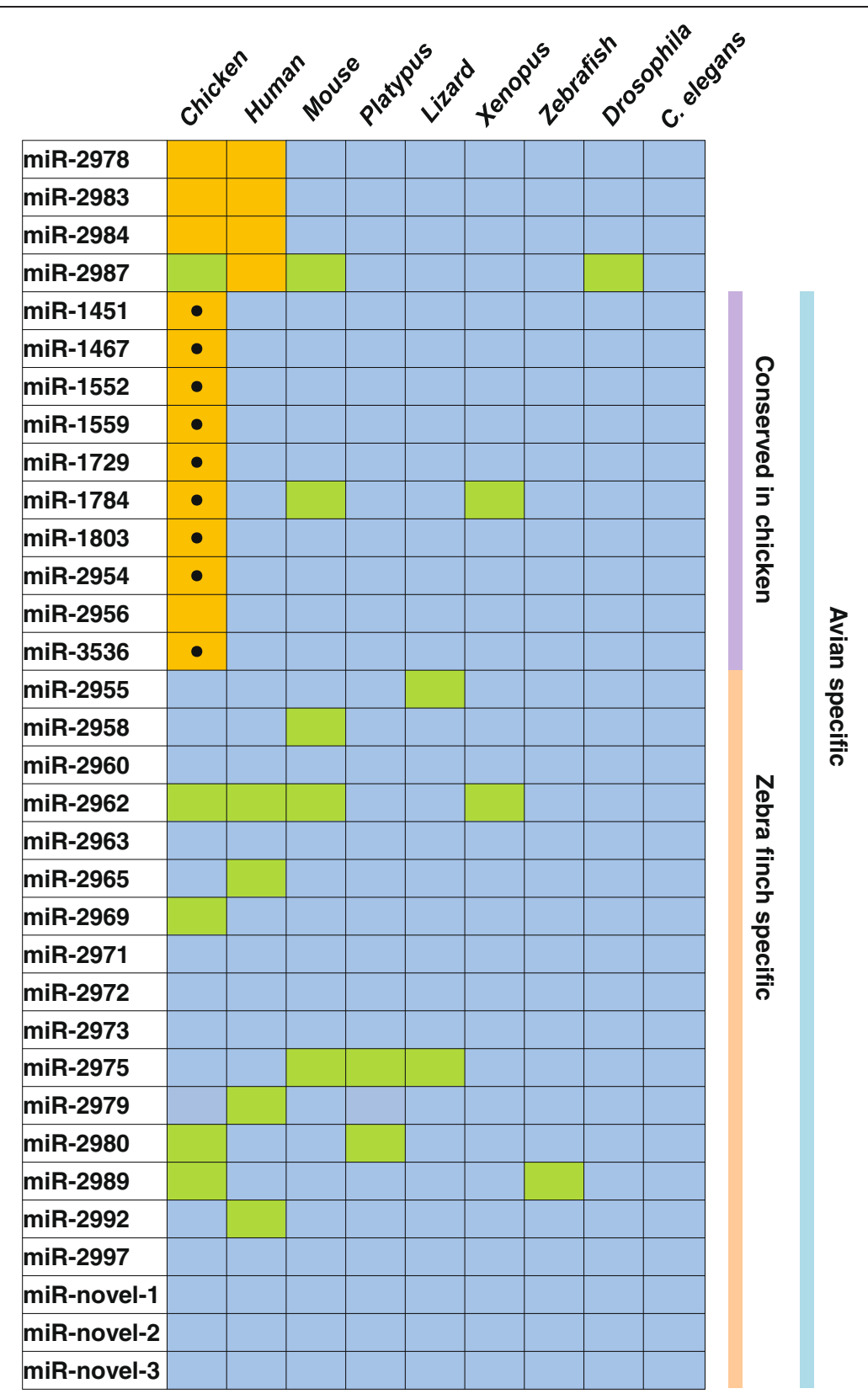

Figure 2 Conservation status of avian-specific miRNAs in nine animal species. The orange color indicates miRNA sequence homologs with predicted hairpin-like structures were found in a genome by our analysis. Black dots indicate homolog miRNAs are already recorded in miRBase as known miRNAs. Green color indicates mature miRNA sequences, but not precursor sequences, were found in a genome, and they were not counted as having homologs in other genomes. Blue is the background. Note, sequence homologs for miR-2978, miR-2983, miR-2984, miR-2987, and miR-2956 were found in the chicken genome, but they were not recorded in miRBase, so they didn't get a black dot. We reported zebra finch specific miRNAs (miR-2955 through miR-2997) previously [32], and they are already in miRBase, so they were not considered to be novel miRNAs.

\section{miRNA*}

We found 150 miRNA* sequences for 131 mature miRNAs (Additional file 5). For miRNAs encoded by multiple genomic loci, a single mature sequence can have more than one star sequence, presumably originating from different precursors. For example, three different star sequences were detected for tgu-miR-7, which has three genomic loci. These three tgu-miR-7* sequences had different read counts, indicating that the three $t g u-m i R-7$ genes were transcribed with different promoter activities and/or different efficacies of precursor processing (Additional file 6). Most of the star sequences were detected at considerably lower levels compared to their respective dominant strands. Yet, for 
16 miRNAs, both strands were detected at comparable levels (< 10 fold differences, Additional file 7), suggesting that both strands may function as mature miRNAs. The relative read counts for the two strands of these miRNAs were similar across the four tissues examined (Additional file 5), indicating that the mechanisms retaining both strands were tissue independent. Interestingly, for several miRNAs (e.g., tgu-miR-142, tgumiR-214, and tgu-miR-455), both strands have been detected at similar levels in mice and chickens as well $[12,38]$, suggesting a functional conservation of these star sequences in vertebrates.

\section{miRNAs generated by atypical biogenesis pathways}

Mirtrons are a special group of miRNAs that are derived from short intronic sequences by splicing machinery rather than by Drosha cleavage [39-41]. Recently, Glazov and colleagues reported the identification of 12 mirtrons in chickens [38]. We did not, however, find sequence homologs of any of the chicken mirtrons in our miRNA set, nor did we find any miRNAs mapping to either boundaries of a short intron. This discrepancy might be partly explained by the fact that the chicken mirtrons were detected in embryos and exhibited low copy numbers [38], whereas our miRNAs were from adult tissues. Alternatively, it may simply be a consequence of the incomplete annotation of intronic regions in the zebra finch genome.

miR-451 is a vertebrate miRNA whose maturation depends on Ago2 cleavage rather than the common Dicer pathway $[42,43]$. We found that tgu-miR-451 was located on chromosome 19, about 100 nt downstream from miR-144, and the mature and precursor sequences of tgu-miR-451 were highly conserved with those in chickens, mice, and humans. Similar to its mammalian counterparts, the $5^{\prime}$-end of tgu-miR-451 was well defined, whereas its $3^{\prime}$-end was highly variable, extending into the loop region of the hairpin structure, presumably reflecting imprecise cleavage by Ago2 (Additional file 8).

\section{miRNA expression patterns in tissues}

We next examined the expression patterns of zebra finch miRNAs in brain, heart, liver, and muscle. Overall, the brain displayed the most diverse miRNA expression and had the largest number of tissue-enriched miRNAs (Figure 3A). About $17 \%$ of all miRNAs (32 of 193) showed enriched expression in a single tissue, and two thirds of them (20 of 32) were highly enriched in brain based on combined read counts from males and females (Figure 3A and 3B). However, the two most abundantly expressed miRNAs (miR-1 expressed in heart and muscle and miR-499 in heart) showed little or no expression in brain. Since the sequences of many miRNAs are conserved through evolution, we asked whether the tissue-enriched miRNA expression patterns were also conserved. To this end, we analyzed previously published data from human, mouse, and sea slug (Aplysia) $[10,19]$. Seven miRNAs (miR-9, miR-124, miR-137, mir153, miR129, mir-218, and miR-138b) showed brainenriched expression in both humans and zebra finches, and miR-124, miR-137, miR-153, and miR-34b have also been detected in the Aplysia nervous system (Table 1). We validated the conservation of tissue expression patterns of five miRNAs in human, mouse, and zebra finch by Northern blot analysis (Figure $3 \mathrm{C}$ ).

miRNAs within a genomic cluster tend to exhibit parallel relative expression patterns across tissues, probably reflecting their shared promoter and tissue specific cistronic transcriptional control. However, the expression abundance of different members of a cluster in a given tissue can be drastically different. For example, miR$133 \mathrm{a}$ and miR-1 of the $133 a(2)$ cluster were both expressed in heart and muscle, but the expression of miR-1 was about 10 times higher than that of miR-133a in each of these tissues. This difference in expression levels between members was characteristic of many genomic clusters (Additional file 9), suggesting that a precursor specific event during the miRNA maturation process might regulate the expression levels of individual miRNAs in a genomic cluster.

\section{Sequence variations}

Taking advantage of the large sequence dataset, we analyzed miRNA sequence variations. We classified miRNA isoforms into three major groups: length variations, untemplated terminal nucleotide additions, and internal substitutions (Figure 4A). The length variants accounted for $25 \%$ of the total miRNA reads, a majority of which (> 80\%) were 3 ' variants (Figure $4 \mathrm{~A})$. This is in good agreement with observations in other species, further supporting the notion that precision at cleavage events at the 5 -termini is necessary to protect the seed sequence at positions $2-8$ of the mature miRNA $[12,44,45]$. Nonetheless, the read numbers of $5^{\prime}$ offset isoforms of several miRNAs were relatively high. For example, miR-124, a brain enriched miRNA, had several 5' offset isoforms, with the combined reads accounting for $15 \%$ of all reads (Figure 4B). Another prominent example was miR-133a, which had two main 5'-isoforms, miR-133a1 and miR-133a2, of which the $5^{\prime}$ terminus of miR-133a2 was shifted 1 nucleotide in the 3 ' direction. These two isoforms were each represented by 77,642 and 98,840 reads, accounting for $32 \%$ and $41 \%$ of the total reads. Interestingly, similar patterns of 5 ' heterogeneity are also observed in mouse miR-124 and miR133a $[12,46]$, indicating that the alternative processing mechanisms giving raise to these isoforms might be evolutionarily conserved. 


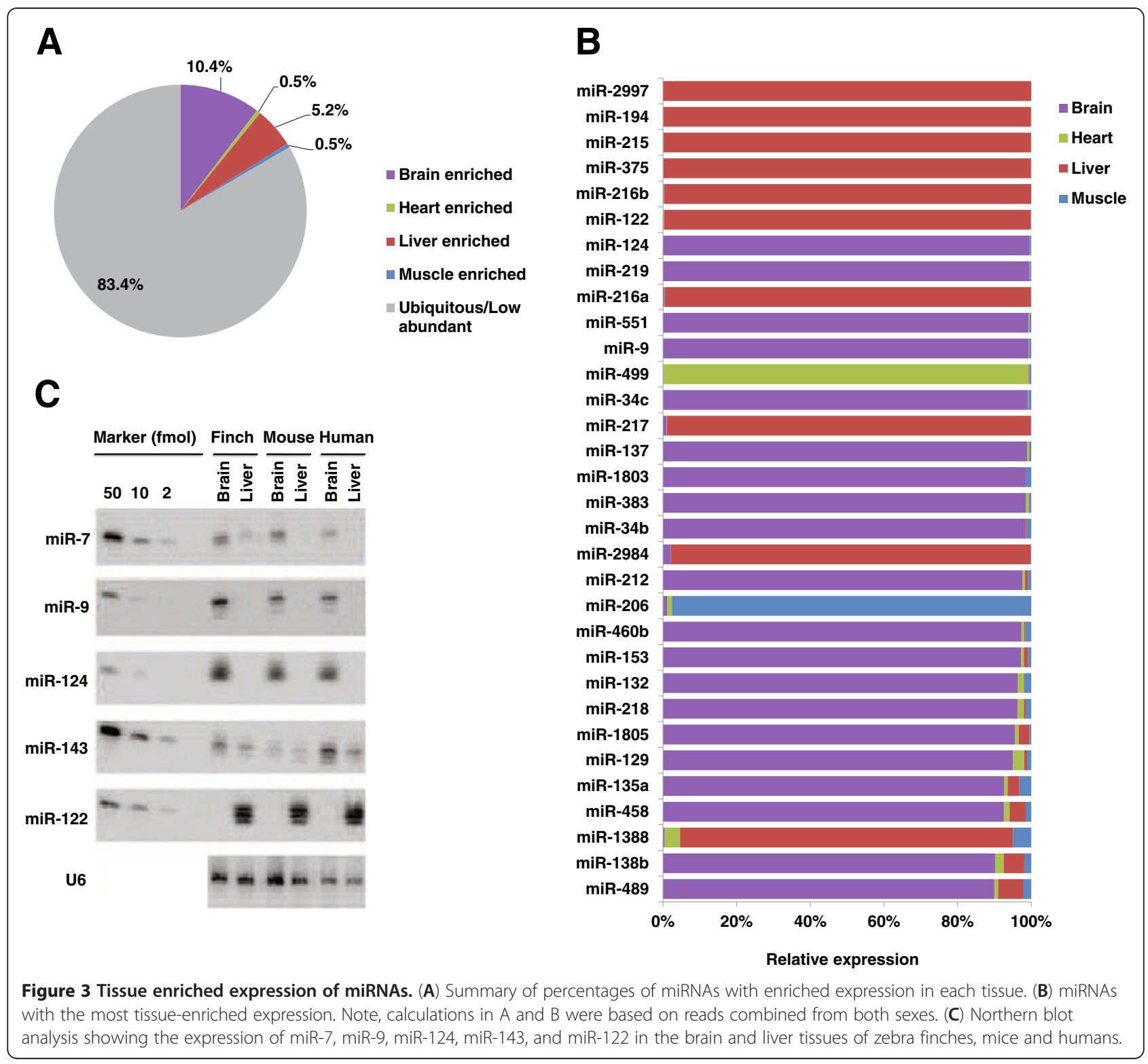

Untemplated nucleotide additions to miRNA 3' ends are observed for many miRNAs in worms, flies, and mammals $[10,12,44,45]$. Similar to those observed in other species, nucleotides most frequently added to zebra finch miRNAs were $U$ and $A$, with the $U$ additions occurring more frequently than the $\mathrm{A}$ additions (66 miRNAs had $\mathrm{U}$ additions and 35 miRNAs had A additions, Additional file 10). For some miRNAs, the extended isoforms outnumbered the canonical forms. For example, miR-456 had 7 fold more extended reads than non-extended reads, and miR-24 had 23,754 extended reads compared to 7,212 non-extended reads (Additional file 10). We also observed that A addition had a greater tendency to occur on $5 \mathrm{p}$ arms, occurring 26 times on $5 p$ arms compared to 9 on $3 p$ arms, while
$\mathrm{U}$ addition occurred slightly more often on $3 p$ arms, occurring 36 times on $3 p$ arms compared to 30 on $5 p$ arms. Compared with previously published data, it appeared that patterns of untemplated extension of some miRNAs were conserved among multiple vertebrate species. For example, the A extensions of miR-99 and miR-101 and the U extensions of miR-15a, miR-24, miR-106, miR-124, and miR-425-5p were observed in zebra finch, human, and mouse $[13,46]$.

In mammals, miRNA editing events in which adenosine is converted to inosine by adenosine deaminases (ADARs) are observed for several miRNAs; the resultant inosine is detected in sequencing as an A-to-G conversion $[10,12,47-49]$. Among our data, however, the A-to-G change was found in just $\sim 0.3 \%$ of total miRNA reads in 


\begin{tabular}{|c|c|c|c|c|c|}
\hline \multirow[t]{2}{*}{ miRNAs } & \multirow{2}{*}{$\begin{array}{l}\text { Total } \\
\text { reads }\end{array}$} & \multirow{2}{*}{$\begin{array}{c}\text { Reads in } \\
\text { brain }\end{array}$} & \multicolumn{3}{|c|}{ Conservation* } \\
\hline & & & Human & Mouse & Aplysia \\
\hline miR-9 & 357811 & 354947 & $\sqrt{ }$ & $\sqrt{ }$ & \\
\hline miR-124 & 111628 & 111170 & $\sqrt{ }$ & $\sqrt{ }$ & $\sqrt{ }$ \\
\hline miR-137 & 28008 & 27599 & $\sqrt{ }$ & $\sqrt{ }$ & $\sqrt{ }$ \\
\hline miR-153 & 16599 & 16188 & $\sqrt{ }$ & & $\sqrt{ }$ \\
\hline miR-212 & 8361 & 8168 & & & \\
\hline miR-135a & 7451 & 7053 & & & \\
\hline miR-219 & 7228 & 7198 & & & \\
\hline miR-132 & 6496 & 6172 & & & \\
\hline miR-34b & 6401 & 6322 & & & $\sqrt{ }$ \\
\hline miR-129 & 5909 & 5535 & $\sqrt{ }$ & & \\
\hline miR-551 & 4992 & 4949 & & & \\
\hline miR-218 & 4221 & 4024 & $\sqrt{ }$ & $\sqrt{ }$ & \\
\hline miR-383 & 3822 & 3743 & & & \\
\hline miR-34c & 3628 & 3593 & & & \\
\hline miR-460b & 1447 & 1402 & & & \\
\hline miR-458 & 1013 & 958 & & & \\
\hline miR-138b & 386 & 358 & $\sqrt{ }$ & $\sqrt{ }$ & \\
\hline miR-1805 & 306 & 297 & & & \\
\hline miR-489 & 304 & 285 & & & \\
\hline miR-1803 & 264 & 261 & & & \\
\hline
\end{tabular}

* $\sqrt{ }$ indicates brain-enriched expression in the examined species.

the brain library and in all 8 libraries combined. This frequency does not differ significantly from the sequencing error rate observed among our synthetic internal control sequences spiked into library preparations $(<0.5 \%)$. We also searched for A-to-I editing events at specific sites in individual miRNAs. Using a criterion of $>5 \%$ mismatch frequency, only tgu-miR-24 exhibited significant A-to-G change at the 15th position of the mature sequence. This change occurred in all tissues, but had the highest frequency in brain ( $9 \%$, Additional file 11$)$. We next examined all miRNAs that had any internal nucleotide change. Thirty-two miRNAs displayed internal nucleotide changes with a frequency $>5 \%$. Nucleotide changes at the $\mathrm{U}$ position within a GGU motif were the most frequent substitution (25 out of the 32 ), and it appeared that substitution with any of the other three nucleotides was permissible (Figure 4C). For several miRNAs in this group, the GGU motifs occurred within the seed sequence (Table 2); thus, substitution of the $U$ nucleotide could potentially alter miRNA targeting specificity (Table 2).

\section{Male-biased expression and targeting of miR-2954}

miR-2954 is a recently identified avian miRNA, which has a single genomic locus on the zebra finch $\mathrm{Z}$ chromosome
[32,35]. We did not detect it in the chicken genome assembly (version galGal3) or in the genomes of other animal species (Figure 2). However, we found the mature miR2954 sequence among chicken ESTs, and its expression in chicken embryo is detected by Northern blot analysis [50]. Tgu-miR-2954 was expressed in a sex-biased manner in all four examined tissues; and its expression was significantly higher in male tissues than in female tissues (Additional file 2). We validated this expression pattern by Northern blot analysis and quantitative real-time PCR (qRT-PCR) (Figure 5A, B, and C). Our original sequencing samples did not include ovary and testis; however, using qRT-PCR, we found that the expression of miR2954 was 3-fold higher in testis than in ovary (Figure 5C). In addition to miR-2954, six other miRNAs (miR-23b, miR-24, miR-27b, miR-122, miR-2973, and miR-2992) were encoded solely by the $Z$ chromosome, and most of them showed slightly higher expression in male tissues than in female tissues (Figure 5D). The one exception was miR-122, which was expressed at a higher level in heart in females than in males. Several miRNAs (miR-7, miR-9, miR-101, and miR-204) that are encoded by both the $\mathrm{Z}$ and the $\mathrm{A}$ chromosomes did not exhibit consistent sex-biased expression (Additional file 12).

We next performed target prediction analysis using the TargetScan software package [51]. Target analysis predicted approximately 100 putative tgu-miR-2954 target genes, with a strong bias towards $\mathrm{Z}$ chromosome-encoded genes (27 of 100, $P<1 e-15$, Fisher's exact test) (Figure 6A). A similar pattern was also observed for chicken miR-2954, for which 70 of the 403 putative targets were $\mathrm{Z}$ chromosome encoded $(17.4 \%, P<2.2 e-16$, Fisher's exact test) (Figure 6B), suggesting that the functional preference of miR-2954 for Z-linked genes is conserved among avians. A significantly larger number of miR-2954 target genes were predicted in chickens (WASHUC2) than in zebra finches, probably reflecting the incomplete annotation of the 3'-UTR sequences in the current zebra finch genome assembly (taeGut3.2.4). In contrast, no enrichment of $\mathrm{Z}$ chromosome-encoded target genes was found for other zebra finch $\mathrm{Z}$ chromosome-encoded miRNAs such as miR-27b and miR-122 (Figure 6C). Among the putative tgu-miR-2954 targets were several Z-linked genes encoding proteins with particular functions in the nervous system, including $\mathrm{Ca}^{2+} /$ calmodulin-dependent protein kinase IV (CaMKIV), SCAMP1, and SMARCA2. Genes encoding proteins in the guanine nucleotide exchange signaling pathways (e.g., RICTOR, DelGEF-interacting protein 1, Brefeldin A-inhibited guanine nucleotide-exchange protein 3, RAB40C, developmentally-regulated GTP-binding protein 2, and MCF2) were prominently represented as well. In addition, many putative miR-2954 targets were key regulators of transcription, including TLE4, GPBP1, PSIP1, SMARCA2, HMGB1, TNIP3, ZCCHC4, 


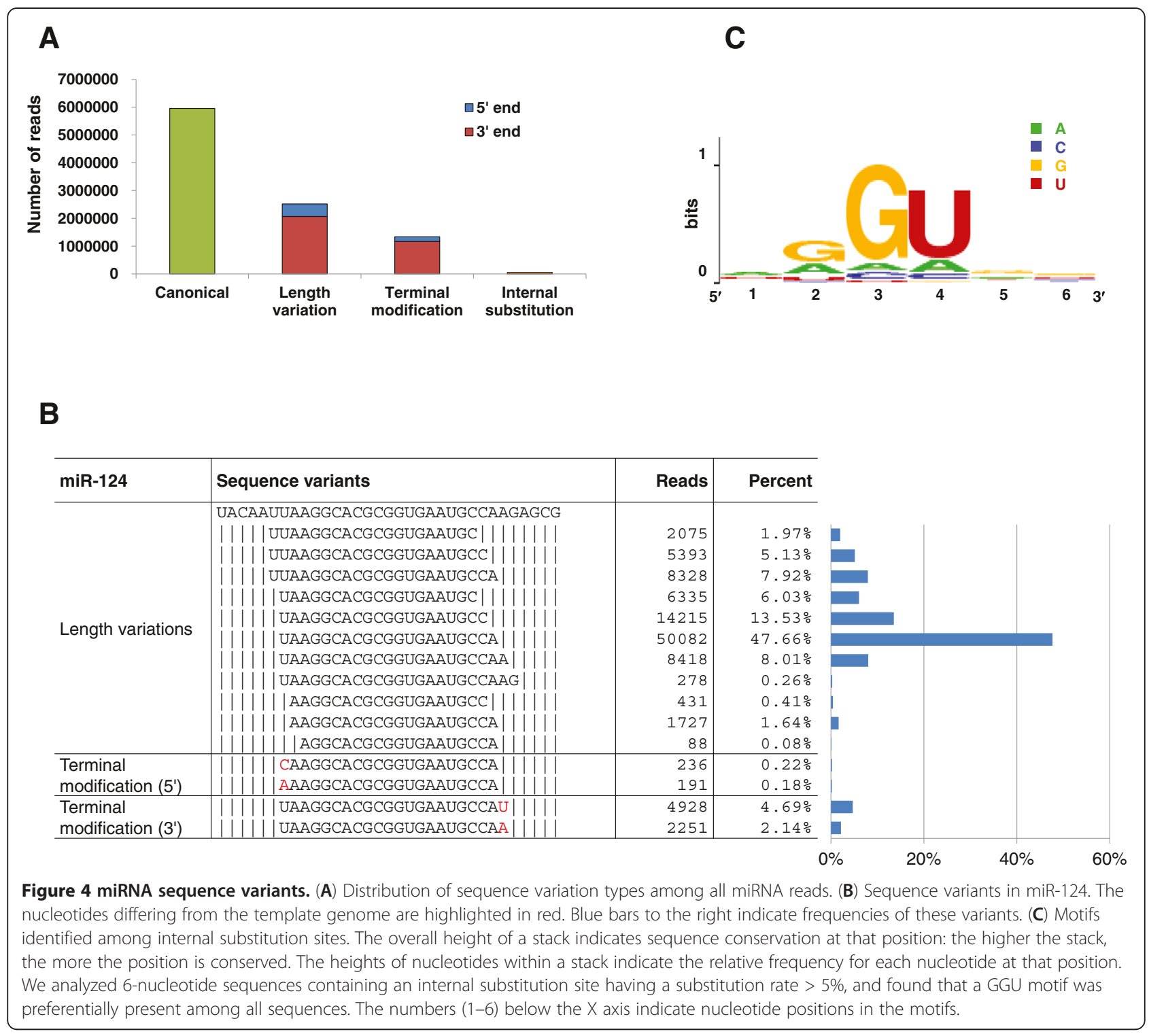

EAPP, RQCD1, MLF1, STAT3, ATF6, EZH1, and DEDD (Additional file 13).

\section{Discussion}

Here we report a comprehensive annotation and analysis of 193 zebra finch miRNAs expressed in four different tissues of both sexes. While the majority of the 193 identified miRNAs are evolutionarily conserved, relatively large fractions of them are zebra finch-specific (10\%) or avian-specific (15\%). The list of zebra finch specific miRNAs may be even longer, as approximately $40 \%$ of the total sequence reads obtained from our small RNA libraries failed to map to the genome, probably due to gaps in the current genome assembly. Conversely, genomes of other species that we searched may have gaps as well, thus we cannot rule out the possibility that some avian and/or zebra finch specific miRNAs described here may have undiscovered homologs in other species. Several zebra finch specific miRNAs showed tissue specific expression. For example, miR2963 was detected in the brain, miR-2997 in liver, and the novel miRNA 3 in heart (Additional file 14). However, most of the zebra finch specific miRNAs were expressed at low levels, indicating that their expression is restricted to specific cell types or that they have not yet been widely incorporated into gene regulation networks. Further investigation will be needed to determine if these avian or zebra finch specific miRNAs play roles in zebra finch specific features.

Recently, Gunaratne et al. reported the annotation of 155 miRNAs expressed in the auditory forebrain region of zebra finches [35]. Among these miRNAs, 140 are 
Table 2 miRNAs having substitutions at the GGU motif

\begin{tabular}{|c|c|c|c|}
\hline miRNAs & Canonical sequence* & Modified reads & Percentage \\
\hline tgu-let-7a & UGAGGUAGUAGGUUGUAUAGUU & 11500 & $5.5 \%$ \\
\hline tgu-let-7b & UGAGGUAGUAGGUUGUGUGGUU & 1629 & $6.7 \%$ \\
\hline tgu-let-7c & UGAGGUAGUAGGUUGUAUGGUU & 1556 & $5.2 \%$ \\
\hline tgu-let-7d & AGAGGUAGUAGGUUGCAUAGUU & 395 & $5.5 \%$ \\
\hline tgu-let-7e & UGAGGUAGUAGAUUGAAUAGUU & 3864 & $5.2 \%$ \\
\hline tgu-let-7f & UGAGGUAGUAGAUUGUAUAGUU & 12490 & $5.1 \%$ \\
\hline tgu-let-7i & UGAGGUAGUAGUUUGUGCUGUU & 3792 & $8.2 \%$ \\
\hline tgu-miR-122 & UGGAGUGUGACAAUGGUGUUUG & 6211 & $14.0 \%$ \\
\hline tgu-miR-133b & UUGGUCCCCUUCAACCAGCUAU & 18 & $5.1 \%$ \\
\hline tgu-miR-140 & ACCACAGGGUAGAACCACGGAC & 674 & $5.0 \%$ \\
\hline tgu-miR-15a & UAGCAGCACAUAAUGGUUUGU & 382 & $5.4 \%$ \\
\hline tgu-miR-15c & UAGCAGCACAUCAUGGUUUGU & 187 & $5.4 \%$ \\
\hline tgu-miR-181b & AACAUUCAUUGCUGUCGGUGGGU & 254 & $6.2 \%$ \\
\hline tgu-miR-183 & UAUGGCACUGGUAGAAUUCACU & 5 & $5.1 \%$ \\
\hline tgu-miR-18a & UAAGGUGCAUCUAGUGCAGAUA & 228 & $12.5 \%$ \\
\hline tgu-miR-18b & UAAGGUGCAUCUAGUGCAGUU & 7 & $5.0 \%$ \\
\hline tgu-miR-196 & UAGGUAGUUUCAUGUUGUUGGG & 8 & $10.0 \%$ \\
\hline tgu-miR-221 & AGCUACAUUGUCUGCUGGGUUUC & 2636 & $6.4 \%$ \\
\hline tgu-miR-222 & AGCUACAUCUGGCUACUGGGUCUC & 1413 & $6.5 \%$ \\
\hline tgu-miR-2970 & GACAGUCAGCAGUUGGUCUGG & 219 & $11.7 \%$ \\
\hline tgu-miR-363 & AAUUGCACGGUAUCCAUCUGU & 83 & $6.8 \%$ \\
\hline tgu-miR-383 & CAGAUCAGAAGGUGAUUGUGGC & 101 & $7.3 \%$ \\
\hline tgu-miR-456 & CAGGCUGGUUAGAUGGUUGUC & 34 & $7.8 \%$ \\
\hline tgu-miR-458 & AUAGCUCUUGGAAUGGUUCUGC & 31 & $5.1 \%$ \\
\hline tgu-miR-551 & GCGACCCAUACUUGGUUUCAG & 69 & $5.0 \%$ \\
\hline
\end{tabular}

*The seed regions (2-8th nucleotides) are underlined. The GGU motifs are marked in boldface.

present in our set of miRNAs. Our dataset contains an additional 53 miRNAs, many of which show tissue specific expression in heart, liver, and muscle (Additional files 2 and 15). This is not unexpected as we sequenced four tissues, whereas only auditory forebrain was sequenced by Gunaratne et al. Although the source materials used in the two studies were different and the criteria for miRNA annotation were slightly different, the large overlap between the two datasets provides additional confidence with respect to the identification of these miRNAs. Combining the two sets of data brings the total number of zebra finch miRNAs to 208. Many of these miRNAs show enriched expression in the brain, including several (miR-25, miR-192, miR-124, miR-129, and miR-92) that are regulated in the auditory forebrain by song exposure [35], indicating that miRNAs may play important roles in song behavior and neural plasticity.

\section{miRNA sequence heterogeneity}

We did not observe significant A-to-I editing in mature zebra finch miRNAs except for miR-24. Notably, several mammalian miRNAs with well-characterized A-to-I editing sites (e.g., miR-376a, miR-376c, miR-379, miR381, miR-411, miR-421, and miR-589, [12,48,49]) do not appear to have homologs in zebra finches, hinting that A-to-I editing might be more restricted to mammals. Instead, we observed internal nucleotide changes at the uridine of a GGU motif occurring at a relative high frequency. Our average base call sequencing error rate was $<0.5 \%$ (calculated based on spiked-in synthetic internal controls), comparable to sequencing error rates observed by others [49]. The rate of observed nucleotide changes at the GGU motif was as high as $14 \%$ (in miR-22). Thus it is unlikely that these changes were due to sequencing errors. We also examined each genomic locus for miRNAs known to have multiple genomic loci, and ruled out the possibility that they were due to genetic variations. We cannot rule out the possibility that these changes were due to single nucleotide polymorphisms (SNPs), as we currently know very little about SNPs in zebra finches. However, we would expect that the rate of nucleotide changes generated by SNPs would be much 


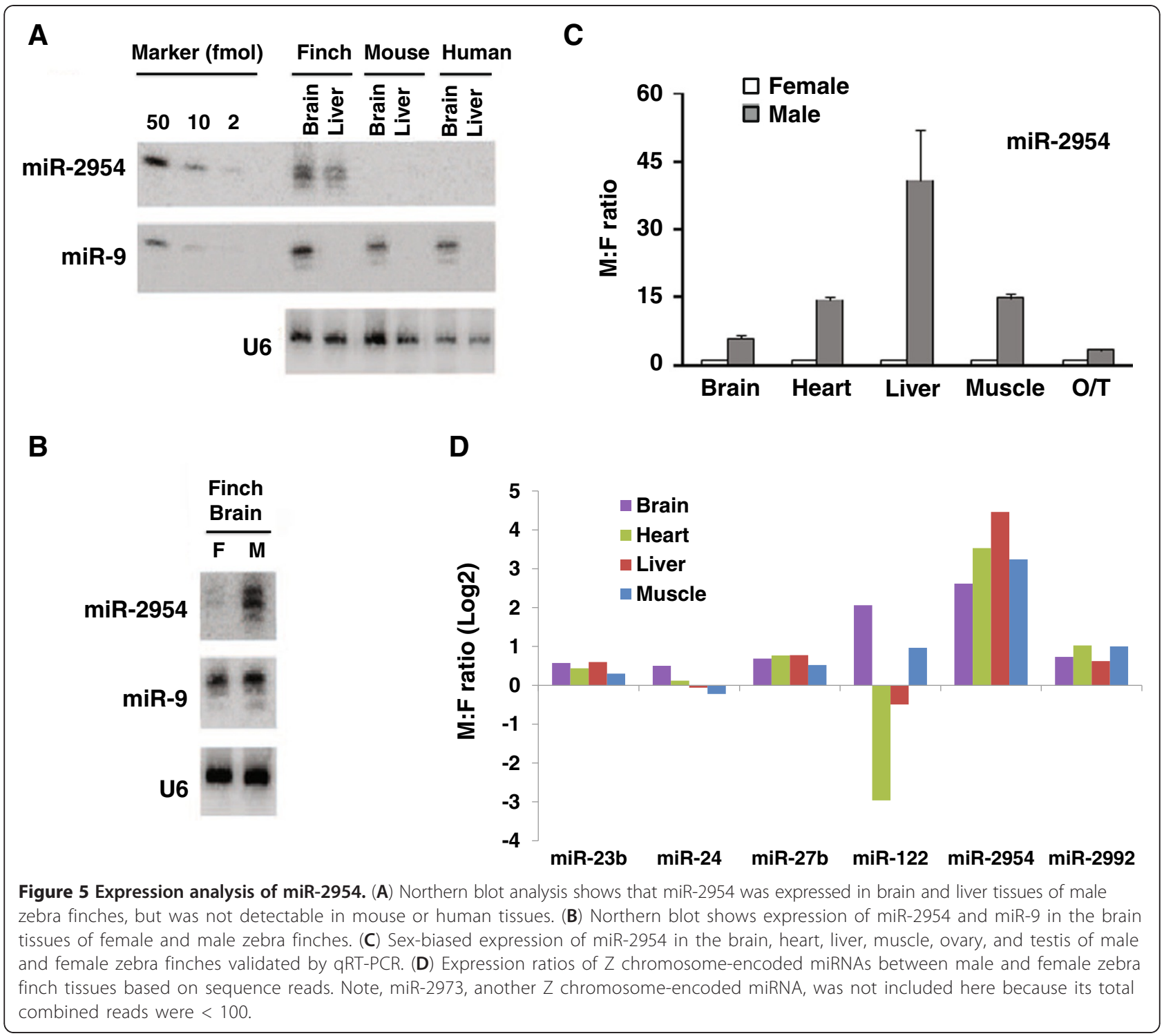

higher than we observed. To our knowledge, internal nucleotide changes at the uridine of a GGU motif has not been reported previously. Some of the GGU motifs are within seed sequences of mature miRNAs (e.g., members of the let-7 family). Nucleotide changes at these sites can change miRNA binding specificity, and subsequently, impact gene expression and related cellular processes. Further analysis will bring better understandings of biosynthesis mechanisms and potential biological functions of this type of internal nucleotide change.

Other types of sequence heterogeneity including untemplated 3' terminal extensions and length variations are far more prevalent than internal nucleotide changes. miRNA isoforms with 3 ' untemplated extensions account for $\sim 15 \%$ of all miRNA reads. miRNA 3' untemplated A or $\mathrm{U}$ extensions have been widely reported by others in multiple animal species [12,13,52-56]. These events seem to be miRNA specific, as some miRNAs are more frequently extended than others. Interestingly, many of the zebra finch miRNAs showing high frequencies of $3^{\prime}$ untemplated extensions are also frequently extended in other species (Additional file 10, [11,57,58]), hinting that mechanisms governing the extension events and their potential functions are likely to be conserved. Although investigation of $3^{\prime}$ untemplated extension is still at an early stage, 3 ' extension appears to be biologically regulated [11] and to affect biological function. Emerging evidence suggests that 3 ' untemplated extensions provide a posttranscriptional mechanism to regulate miRNA stability and efficiency of target repression [57-59].

miR-2954 as a sex specific gene regulator in avian species Despite extensive searching, we did not find sequence homologs of miR-2954 outside of the avian taxon. An 

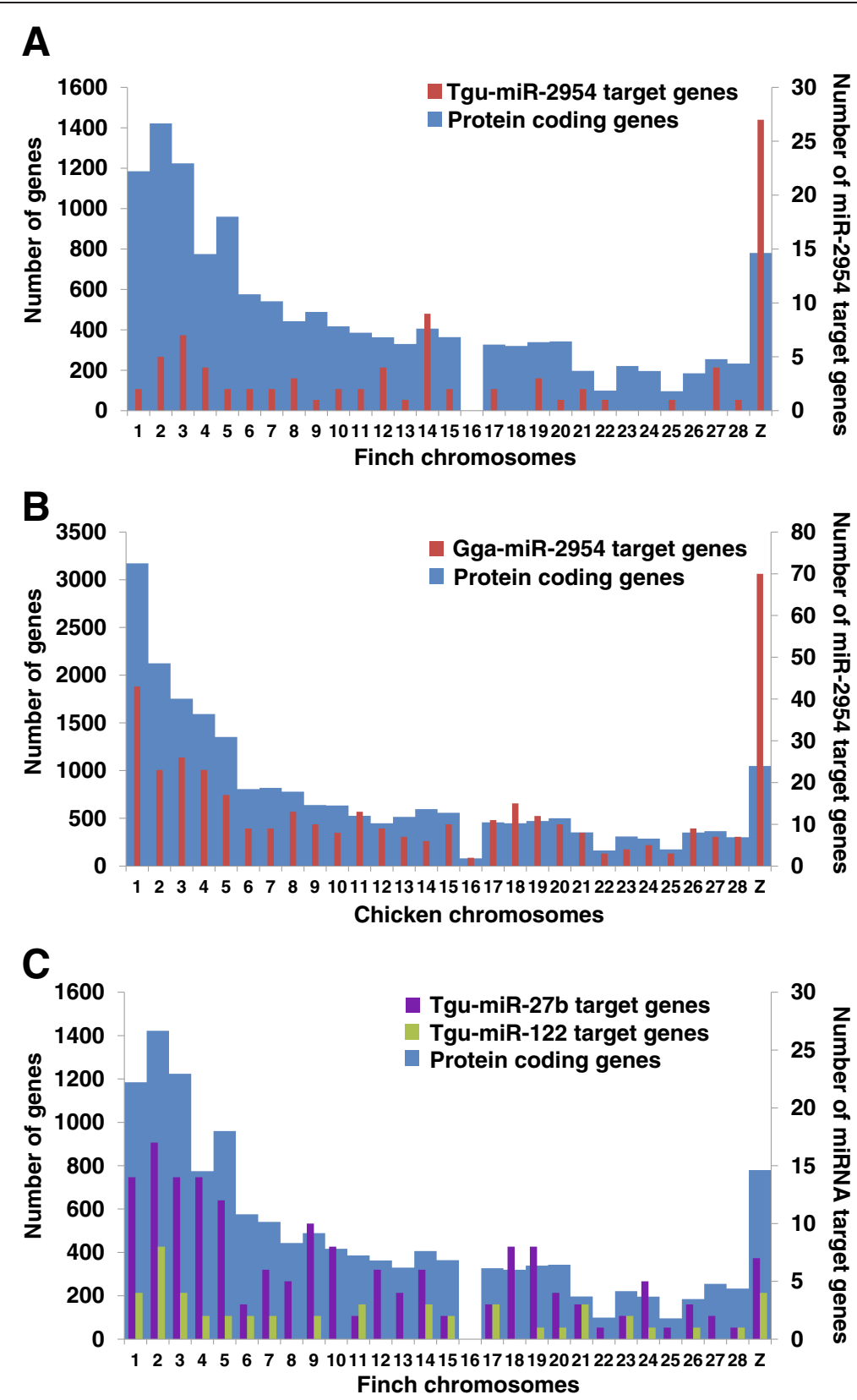

Figure 6 Z chromosome distribution bias of predicted targets for miR-2954. (A) Distribution of predicted targets for miR-2954 among zebra finch chromosomes. (B) Distribution of predicted targets for miR-2954 among chicken chromosomes. Note, in both zebra finches and chickens, miR-2954 targets exhibited a strong bias towards Z chromosome-encoded genes. (C) Distribution of predicted targets for miR-27b and miR-122, two other $Z$ chromosome-encoded miRNAs, among zebra finch chromosomes. The distributions of protein coding genes among zebra finch and chicken chromosomes are shown in blue color.

extensive search among transcripts of crocodile and 11 bird species conducted by Gunaratne et al. found miR2954 in several bird species but not in crocodile [35]. Given that the genomes and transcriptomes searched in these studies are not complete, we cannot rule out the possibility that miR-2954 has as yet unidentified homologs in other species. However, its expression in chickens and zebra finches is now supported by three independent studies including ours $[32,35,50]$. The male-biased expression of miR-2954 in chicken embryos [50] and in various zebra finch tissues suggests that it plays a role in sexually dimorphic animal development and function in avian species.

Animals adapt different dosage compensation mechanisms to balance the expression of sex chromosome genes between the two sexes and to balance the expression 
between sex chromosomal genes and autosomal genes $[26,60]$. In avian species, females are heterogametic with one $\mathrm{Z}$ and one $\mathrm{W}$ chromosome, and males are homogametic with two $\mathrm{Z}$ chromosomes. Both the chicken and zebra finch lack a chromosome-wide dosage compensation mechanism, and many $\mathrm{Z}$ chromosome genes exhibit higher expression in males than in females [61-67]. While the male to female (M:F) expression ratios of most Z-linked miRNAs are close to 2, reflecting the copy number relationship, miR-2954 exhibits M:F expression ratios ranging from 6 to 20 (based on read counts) or 6 to 40 (based on qRT-PCR) in various tissues, which cannot be explained by the 2:1 difference in gene copy numbers. Clearly, additional male-biased factors contribute to the regulation of miR-2954 expression in various tissues.

In both chickens and zebra finches, dosage compensation can occur locally in a gene-specific manner [65-67]. In chickens, this is exemplified by dosage compensation mediated by the MHM (male hypermethylated) locus on nearby genes on the $\mathrm{Z}$ chromosome $[26,68,69]$. However, the zebra finch genome does not appear to contain the MHM locus [66], suggesting the existence of other sex specific dosage compensation mechanisms. The male-biased expression of miR-2954 and its preferential targeting of Z-chromosome genes may provide a novel dosage compensation mechanism at the posttranscriptional level. A miRNA-mediated gene-specific mechanism for sex chromosome gene regulation would offer flexibility in response to specific developmental and functional needs.

Both song behavior and the underlying neural circuit are highly sexually dimorphic in zebra finches. The song system nuclei HVC [70] and the robust nucleus of the arcopallium (RA), which control motor patterns of song, are considerably larger in males than in females, and Area-X, a forebrain nucleus required for song learning, is a large nucleus in adult males but is almost invisible in adult females $[27,71]$. The gene regulation network underlying this structural and functional sexual dimorphism is not clear. Among the putative targets of miR-2954, SCAMP1, a Z chromosome encoded synaptic vesicle associated protein gene, is known to be expressed at higher levels in HVC and RA in male zebra finches than in females [72]. Recently, Gunaratne et al. reported that miR-2954 is regulated by hearing songs in the auditory forebrain of zebra finches [35], suggesting that in the context of song behavior this miRNA plays important roles in relaying physiological changes to changes in gene expression. Further analysis of miR-2954 expression in the song control circuits, and validation of its target genes will provide a better understanding of its roles in the sexually dimorphic structural and functional development of the zebra finch brain.

\section{Conclusions}

Our results provide a comprehensive miRNA expression atlas of brain, heart, liver, and muscle tissues of both male and female zebra finches. These data significantly enlarge the zebra finch miRNA repertoire, and will serve as a valuable resource for comparative and functional studies for the scientific community. In addition, we report a GGU motif as a potential site for miRNA internal substitution. We also describe male-biased expression of tgu-miR-2954, as well as its Z chromosome biased target relationship, which may point to a novel avian specific dosage compensation mechanism.

\section{Methods}

\section{Library preparation and sequencing}

Four tissues (heart, liver, muscle, and whole brain) from adult male and female zebra finches were collected, and total RNA was isolated using the Trizol method. The RNAs were used for library construction. Briefly, in a total reaction volume of $20 \mu \mathrm{l}, 2 \mu \mathrm{g}$ total RNA was ligated to 100 pmol adenylated $3^{\prime}$ adapter containing a unique pentamer barcode (App-(Barcode)TCGTATGCCGTCT TCTGCTTGT), 1 нg Rnl2(1-249)K227Q (plasmid for expression of recombinant ligase is available at www. addgene.org) in $50 \mathrm{mM}$ Tris- $\mathrm{HCl}, \mathrm{pH} 7.6 ; 10 \mathrm{mM}$ $\mathrm{MgCl}_{2} ; 10 \mathrm{mM}$ 2-mercaptoethanol; $0.1 \mathrm{mg} / \mathrm{ml}$ acetylated BSA (Sigma, St. Louis, MO), and 15\% DMSO for 16 hours on ice. Following $3^{\prime}$ adapter ligation, 20 bar-coded samples were pooled and products were purified on a $15 \%$ denaturing polyacrylamide gel. Small RNAs, measuring 45-50 nt in length, were excised from the gel, eluted, and ligated to 100 pmol $5^{\prime}$ oligoribonucleotide adapter (GUUCAGAGUUCUACAGUCCGACGAUC) in a $20 \mu \mathrm{l}$ reaction volume using $1 \mu \mathrm{g}$ Rnl1 RNA Ligase in $50 \mathrm{mM}$ Tris- $\mathrm{HCl}$, pH 7.6; $10 \mathrm{mM} \quad \mathrm{MgCl}_{2} ; 10 \mathrm{mM}$ 2mercaptoethanol; $0.2 \mathrm{mg} / \mathrm{ml}$ acetylated BSA; $0.2 \mathrm{mM}$ ATP, and $15 \%$ DMSO for $1 \mathrm{~h}$ at $37^{\circ} \mathrm{C}$. Ligated small RNAs were purified on a $12 \%$ polyacrylamide gel, reverse transcribed using SuperScript III Reverse Transcriptase (Invitrogen, Carlsbad, CA), and amplified by PCR using appropriate primers (forward primer: AATGATACGGC GACCACCGACAGGTTCAGAGTTCTACAGTCCGA; RT and reverse primer: CAAGCAGAAGACGGCATA CGA). A total of eight libraries were barcoded and sequenced in one lane using Illumina GAII.

\section{Sequence analysis and miRNA identification}

After adapter trimming and removal of orphan reads, reads of 18-32 nt in length were kept for further analysis. For miRNA annotations, we compared sequences to known miRNAs (miRBase 17.0, 04/2011), and identical sequences were identified as homology miRNAs. Sequences were mapped to the zebra finch genome (Ensembl, taeGut3.2.4) permitting no mismatches. Sequences with more 
than 100 genomic loci were excluded from further analysis. Sequences homologous (with at most one mismatch) to known tRNA/rRNA/ncRNA sequences collected from the NCBI GenBank database were classified as tRNAs/rRNAs/ ncRNAs. Small RNAs derived from repeat region/transposable elements were identified by screening the zebra finch genome using the RepeatMasker software. The remaining sequences were used for new miRNA candidate prediction. Flanking genomic sequences of various lengths (60, 80,100 , and $120 \mathrm{nt}$ ) of each small RNA mapping locus were extracted and subjected to analysis by the mFold program (http://mfold.rna.albany.edu/) [36] to predict secondary structures. miRNA candidates were identified using the following criteria: (1) presence of hairpinshaped precursor structures, (2) presence of $>10$ sequence reads, (3) presence of star sequences originated from the opposite stem of the hairpin structure, and (4) precise 5' ends among all sequence variants. In addition, sequences homologous to known miRNAs in miRBase, without the star sequence were also accepted. Because the incompleteness of the current genome precluded unambiguous analysis, 16 miRNAs which did not meet criterion 1 but were homologous to known miRNAs were also accepted.

\section{Sequence conservation analysis}

We searched miRBase for known miRNA homologs in 9 species: chicken (galGal3), human (hg18), mouse (mm9), platypus (ornAna1), lizard (anoCar2), X. tropicalis (xenTro3), zebrafish (danRer7), drosophila (dm3), and C. elegans (ce10). We also compared zebra finch miRNA sequences to the genomes and ESTs (downloaded from the UCSC genome resources) of the same 9 species to search for homologs with at most 1 mismatch to the query sequence and presence of a hairpin shaped precursor structure. Qualified matches were identified as candidate miRNA homologs in the tested species. According to results based on these criteria, zebra finch miRNAs were classified into groups of zebra finch specific, avian specific, conserved in avian, human, and mouse, conserved in vertebrates, and conserved in all tested species.

\section{Tissue specificity}

We normalized the read counts of individual miRNA species to the total miRNA read number in each tissue to obtain an RPM measurement, "Reads Per Million reads". RPM values for each miRNA in all tested tissues were compared. We defined a miRNA as "highly enriched in one tissue" if it had a minimum of 100 reads in all tissues, and its expression in one tissue accounted for $\geq 90 \%$ of all reads in all tissues combined.

\section{miRNA sequence variant identification}

All sequences passing the qualification filter were used for miRNA variant analysis. Length variants were selected among sequences matched perfectly to the zebra finch genome by comparing their lengths with the canonical miRNA sequences. For variants containing terminal modifications and internal substitutions, the NCBI Blastn program was used to compare all qualified sequence reads with identified miRNAs. Sequences matching multiple miRNAs were excluded to avoid ambiguity. The criteria used for accepting an internal nucleotide change variant included i) a requirement that the total number of sequence reads of a miRNA including its variants was $>100$, ii) the number of reads with internal changes represented more than $5 \%$ of total reads, and iii) the mismatch occurred at positions at least 2 nucleotides from the 5' and 3' termini. A plot of the motif analysis (Figure 4C) was generated with the WebLogo program (http://weblogo.berkeley.edu/ [73]).

\section{Target gene prediction}

For target gene prediction, the 3' UTR sequences of all zebra finch and chicken genes were extracted from the Ensemble database (taeGut3.2.4 and WASHUC2) using the BioMart tool (www.ensembl.org/biomart/martview). Genes with 3' UTR of less than 10 nt or without 3' UTR were excluded. Since TargetScan (version 6.0, [51]) does not support zebra finch sequences, we made minor modifications to adapt it for target analysis in the zebra finch. Genes containing target sequences within the 3' UTRs that match perfectly to the $2-8 \mathrm{nt}$ of a miRNA sequence were accepted as putative targets. Enrichment of miRNA targets on the $\mathrm{Z}$ chromosome was evaluated by Fisher's exact test.

\section{Northern blot and qRT-PCR}

Total RNAs were extracted from relevant tissues of adult male and female zebra finches with Trizol reagent (Invitrogen). Total RNAs of chicken, human, and mouse were purchased from Ambion. For Northern blot analysis, $20 \mu \mathrm{g}$ RNA from each tissue was separated on $20 \%$ denaturing gel, transferred to a nitrocellulose membrane, and hybridized to relevant miRNA probes labeled with ${ }^{32} \mathrm{P}-\mathrm{dCTP}$ at $65^{\circ} \mathrm{C}$ overnight. Hybridization signals were detected using a phosphorimager. For qRT-PCR, reverse transcription was performed using a custom-designed miR-2954 specific primer and the TaqMan microRNA reverse transcription kit (Applied Biosystems). qRT-PCR was performed in a $20 \mu \mathrm{L}$ reaction volume containing $1.33 \mu \mathrm{L}$ cDNA, $1 \mu \mathrm{L} 20 \times$ custom designed miR-2954 Taqman probe, and the Taqman universal PCR master mix (Applied Biosystems) using conditions of $10 \mathrm{~min}$ at $95^{\circ} \mathrm{C}$, followed by 40 cycles of $15 \mathrm{sec}$ at $90^{\circ} \mathrm{C}, 1 \mathrm{~min}$ at $60^{\circ} \mathrm{C}$. Relative miR-2954 expression was calculated by the $\Delta \Delta \mathrm{Ct}$ method as described [74]. GAPDH was used as an internal control for normalizing miR-2954 expression, as no sex-based differential expression of GAPDH has been detected in zebra finches [65]. All qRTPCR reactions were run in triplicate. 


\section{Additional files}

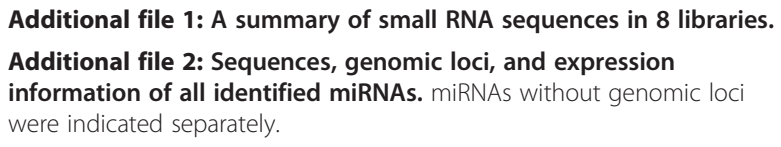

Additional file 3: Conservation status of all identified miRNAs and their expression reads.

Additional file 4: Genomic clusters of zebra finch miRNAs and their conservation status in other species.

Additional file 5: miRNA* $^{*}$ sequences and their expression information.

Additional file 6: Sequences and expression of 3 miR-7*s. Mature miRNAs and corresponding miRNA*s are highlighted by red and blue, respectively.

Additional file 7: miRNA precursors with both $5 p$ and $3 p$ arms producing high abundant mature miRNAs.

Additional file 8: Sequence reads of tgu-miR-451 and precursor structures of the atypically generated miR-451 in zebra finch, mouse, and human.

Additional file 9: Expression patterns of miRNA clusters in the four tissues. (A) A heatmap was plotted according to the log2 transformed normalized reads in each tissue. (B) The relative expression of three miRNA clusters in 4 tissues.

Additional file 10: miRNA variants with untemplated $3^{\prime}$ terminal modifications.

Additional file 11: Summary of internal nucleotide changes observed among miRNA variants. (A) The total sequence reads of each nucleotide change type detected in all tissues. (B) The relative ratio of each nucleotide change type in each tissue sample.

Additional file 12: Expression information of $Z$ chromosomeencoded miRNAs.

Additional file 13: Predicted target genes of tgu-miR-2954.

Additional file 14: Expression information of zebra finch specific miRNAs.

Additional file 15: Comparison with the miRNA set from the Gunaratne et al. study. Fifty-three miRNAs unique in this study are listed in the box.

\section{Additional information}

The three novel zebra finch miRNAs have been assigned miRBase IDs as tgumiR-7643 (novel 1), tgu-miR-7644 (novel 2), and tgu-miR-7645 (novel 3).

\section{Competing interests}

All authors declare that they have no competing interests.

\section{Authors' contributions}

GZL carried out the bioinformatics analysis and participated in writing the paper. MH performed small RNA library construction and sequencing work and participated in writing the paper. MB and GHF helped analyze the data. ZS performed RNA isolation, GRT-PCR, and Northern blot assays. TT participated in designing the work. XCL and XJW designed the study, supervised the experiments and data analysis, and wrote the paper. All authors read and approved the final manuscript.

\section{Acknowledgments}

This study was supported by NIH grant MH081254 (XCL) and grants, 973 2011 CBA01101 from the National Basic Research Program of China and KSCX2-EW-R-0103 from Chinese Academy of Sciences (XJW). MH was supported by the Charles Revson Jr. foundation. We thank Dr. C. Zhao and Genomic Core of the Rockefeller University for the sequencing work. We thank the Washington University Genome Center for sharing the zebra finch genome sequence prior to publication.

\section{Author details}

${ }^{1}$ State Kay Laboratory of Plant Genomics, Institute of Genetics and Developmental Biology, Chinese Academy of Sciences, Beijing 100101, China. ${ }^{2}$ Laboratory of RNA Molecular Biology, The Rockefeller University, New York, NY 10065, USA. ${ }^{3}$ Neuroscience Center of Excellence, Louisiana State University Health Sciences Center, New Orleans, LA 70112, USA.

Received: 7 August 2012 Accepted: 21 December 2012

Published: 26 December 2012

\section{References}

1. Bartel DP: MicroRNAs: target recognition and regulatory functions. Cell 2009, 136(2):215-233.

2. Lee Y, Ahn C, Han J, Choi H, Kim J, Yim J, Lee J, Provost P, Radmark O, Kim S, et al: The nuclear RNase III drosha initiates microRNA processing. Nature 2003, 425(6956):415-419.

3. Gregory RI, Yan KP, Amuthan G, Chendrimada T, Doratotaj B, Cooch N, Shiekhattar R: The microprocessor complex mediates the genesis of microRNAs. Nature 2004, 432(7014):235-240.

4. Han J, Lee $Y$, Yeom KH, Kim YK, Jin H, Kim VN: The drosha-DGCR8 complex in primary microRNA processing. Genes Dev 2004, 18(24):3016-3027.

5. Winter J, Jung S, Keller S, Gregory Rl, Diederichs S: Many roads to maturity: microRNA biogenesis pathways and their regulation. Nat Cell Biol 2009, 11(3):228-234

6. Lund E, Guttinger S, Calado A, Dahlberg JE, Kutay U: Nuclear export of microRNA precursors. Science 2004, 303(5654):95-98.

7. Yi R, Qin Y, Macara IG, Cullen BR: Exportin-5 mediates the nuclear export of pre-microRNAs and short hairpin RNAs. Genes Dev 2003, 17(24):3011-3016.

8. Krol J, Loedige I, Filipowicz W: The widespread regulation of microRNA biogenesis, function and decay. Nat Rev Genet 2010, 11(9):597-610.

9. Yang JS, Phillips MD, Betel D, Mu P, Ventura A, Siepel AC, Chen KC, Lai EC: Widespread regulatory activity of vertebrate microRNA* species. RNA 2011, 17(2):312-326.

10. Landgraf P, Rusu M, Sheridan R, Sewer A, lovino N, Aravin A, Pfeffer S, Rice A, Kamphorst AO, Landthaler $\mathrm{M}$, et al: A mammalian microRNA expression atlas based on small RNA library sequencing. Cell 2007, 129(7):1401-1414.

11. Wyman SK, Knouf EC, Parkin RK, Fritz BR, Lin DW, Dennis LM, Krouse MA, Webster PJ, Tewari M: Post-transcriptional generation of miRNA variants by multiple nucleotidyl transferases contributes to miRNA transcriptome complexity. Genome Res 2011, 21(9):1450-1461.

12. Chiang HR, Schoenfeld LW, Ruby JG, Auyeung VC, Spies N, Baek D Johnston WK, Russ C, Luo S, Babiarz JE, et al: Mammalian microRNAs: experimental evaluation of novel and previously annotated genes. Genes Dev 2010, 24(10):992-1009.

13. Burroughs AM, Ando Y, de Hoon MJ, Tomaru Y, Nishibu T, Ukekawa R, Funakoshi T, Kurokawa T, Suzuki $H$, Hayashizaki $Y$, et al: A comprehensive survey of $3^{\prime}$ animal miRNA modification events and a possible role for $3^{\prime}$ adenylation in modulating miRNA targeting effectiveness. Genome Res 2010, 20(10):1398-1410.

14. Brodersen $P$, Voinnet $O$ : Revisiting the principles of microRNA target recognition and mode of action. Nat Rev Mol Cell Biol 2009, 10(2):141-148

15. Gu S, Jin L, Zhang F, Sarnow P, Kay MA: Biological basis for restriction of microRNA targets to the $3^{\prime}$ untranslated region in mammalian mRNAs. Nat Struct Mol Biol 2009, 16(2):144-150.

16. Hafner M, Landthaler M, Burger L, Khorshid M, Hausser J, Berninger $P$, Rothballer A, Ascano M Jr, Jungkamp AC, Munschauer M, et al: Transcriptome-wide identification of RNA-binding protein and microRNA target sites by PAR-CLIP. Cell 2010, 141(1):129-141.

17. Dannemann M, Nickel B, Lizano E, Burbano HA, Kelso J: Annotation of primate miRNAs by high throughput sequencing of small RNA libraries. BMC Genomics 2012, 13:116.

18. Murchison EP, Kheradpour P, Sachidanandam R, Smith C, Hodges E, Xuan Z, Kellis M, Grutzner F, Stark A, Hannon GJ: Conservation of small RNA pathways in platypus. Genome Res 2008, 18(6):995-1004

19. Rajasethupathy P, Fiumara F, Sheridan R, Betel D, Puthanveettil SV, Russo JJ, Sander C, Tuschl T, Kandel E: Characterization of small RNAs in aplysia reveals a role for miR-124 in constraining synaptic plasticity through CREB. Neuron 2009, 63(6):803-817. 
20. Wan LC, Zhang H, Lu S, Zhang L, Qiu Z, Zhao Y, Zeng QY, Lin J: Transcriptome-wide identification and characterization of miRNAs from pinus densata. BMC Genomics 2012, 13:132.

21. Kozomara A, Griffiths-Jones S: MiRBase: integrating microRNA annotation and deep-sequencing data. Nucleic Acids Res 2011, 39(Database issue):D152-157.

22. Friedman RC, Farh KK, Burge CB, Bartel DP: Most mammalian mRNAs are conserved targets of microRNAs. Genome Res 2009, 19(1):92-105.

23. Bartel DP: MicroRNAs: genomics, biogenesis, mechanism, and function. Cell 2004, 116(2):281-297.

24. Doupe AJ, Kuhl PK: Birdsong and human speech: common themes and mechanisms. Annu Rev Neurosci 1999, 22:567-631.

25. Nottebohm F: The anatomy and timing of vocal learning in birds. In The design of animal communication. Edited by Hauser MD, Konishi M. Cambridge: Mass: MIT Press; 1999:63-110.

26. Arnold AP, Itoh Y, Melamed E: A bird's-eye view of sex chromosome dosage compensation. Annu Rev Genomics Hum Genet 2008, 9:109-127.

27. Nottebohm F, Arnold AP: Sexual dimorphism in vocal control areas of the songbird brain. Science 1976, 194(4261):211-213

28. Mooney R: Neural mechanisms for learned birdsong. Learn Mem 2009, 16(11):655-669.

29. Wada K, Howard JT, McConnell P, Whitney O, Lints T, Rivas MV, Horita H, Patterson MA, White SA, Scharff C, et al: A molecular neuroethological approach for identifying and characterizing a cascade of behaviorally regulated genes. Proc Natl Acad Sci USA 2006, 103(41):15212-15217.

30. Li X, Wang XJ, Tannenhauser J, Podell S, Mukherjee P, Hertel M, Biane J, Masuda S, Nottebohm F, Gaasterland T: Genomic resources for songbird research and their use in characterizing gene expression during brain development. Proc Natl Acad Sci USA 2007, 104(16):6834-6839.

31. Replogle K, Arnold AP, Ball GF, Band M, Bensch S, Brenowitz EA, Dong S, Drnevich J, Ferris M, George JM, et al: The songbird neurogenomics (SoNG) initiative: community-based tools and strategies for study of brain gene function and evolution. BMC Genomics 2008, 9:131

32. Warren WC, Clayton DF, Ellegren H, Arnold AP, Hillier LW, Kunstner A, Searle S, White S, Vilella AJ, Fairley S, et al: The genome of a songbird. Nature 2010, 464(7289):757-762

33. Lovell PV, Clayton DF, Replogle KL, Mello CV: Birdsong "transcriptomics": neurochemical specializations of the oscine song system. PLOS One 2008, 3(10):e3440.

34. Hilliard AT, Miller JE, Fraley ER, Horvath S, White SA: Molecular microcircuitry underlies functional specification in a basal ganglia circuit dedicated to vocal learning. Neuron 2012, 73(3):537-552.

35. Gunaratne $\mathrm{PH}$, Lin YC, Benham AL, Drnevich J, Coarfa C, Tennakoon JB, Creighton CJ, Kim JH, Milosavljevic A, Watson M, et al: Song exposure regulates known and novel microRNAs in the zebra finch auditory forebrain. BMC Genomics 2011, 12(1):277.

36. Zuker M: Mfold web server for nucleic acid folding and hybridization prediction. Nucleic Acids Res 2003, 31(13):3406-3415.

37. Griffiths-Jones S: The microRNA registry. Nucleic Acids Res 2004 32(Database issue):D109-111.

38. Glazov EA, Cottee PA, Barris WC, Moore RJ, Dalrymple BP, Tizard ML: A microRNA catalog of the developing chicken embryo identified by a deep sequencing approach. Genome Res 2008, 18(6):957-964.

39. Okamura K, Hagen JW, Duan H, Tyler DM, Lai EC: The mirtron pathway generates microRNA-class regulatory RNAs in drosophila. Cell 2007, 130(1):89-100

40. Ruby JG, Jan CH, Bartel DP: Intronic microRNA precursors that bypass drosha processing. Nature 2007, 448(7149):83-86.

41. Berezikov E, Chung WJ, Willis J, Cuppen E, Lai EC: Mammalian mirtron genes. Mol Cell 2007, 28(2):328-336

42. Yang JS, Lai EC: Dicer-independent, Ago2-mediated microRNA biogenesis in vertebrates. Cell Cycle 2010, 9(22):4455-4460.

43. Cifuentes D, Xue H, Taylor DW, Patnode H, Mishima Y, Cheloufi S, Ma E, Mane S, Hannon GJ, Lawson ND, et al: A novel miRNA processing pathway independent of dicer requires Argonaute2 catalytic activity. Science 2010, 328(5986):1694-1698.

44. Ruby JG, Jan C, Player C, Axtell MJ, Lee W, Nusbaum C, Ge H, Bartel DP: Large-scale sequencing reveals 21U-RNAs and additional microRNAs and endogenous siRNAs in C. Elegans. Cell 2006, 127(6):1193-1207.

45. Ruby JG, Stark A, Johnston WK, Kellis M, Bartel DP, Lai EC: Evolution, biogenesis, expression, and target predictions of a substantially expanded set of drosophila microRNAs. Genome Res 2007 17(12):1850-1864

46. Zhou H, Arcila ML, Li Z, Lee EJ, Henzler C, Liu J, Rana TM, Kosik KS: Deep annotation of mouse iso-miR and iso-moR variation. Nucleic Acids Res 2012, 40(13):5864-5875.

47. Yang W, Chendrimada TP, Wang Q, Higuchi M, Seeburg PH, Shiekhattar R, Nishikura K: Modulation of microRNA processing and expression through RNA editing by ADAR deaminases. Nat Struct Mol Biol 2006, 13(1):13-21.

48. Kawahara Y, Zinshteyn B, Sethupathy $P$, lizasa $H$, Hatzigeorgiou AG, Nishikura K: Redirection of silencing targets by adenosine-to-inosine editing of miRNAs. Science 2007, 315(5815):1137-1140.

49. Alon S, Mor E, Vigneault F, Church GM, Locatelli F, Galeano F, Gallo A, Shomron N, Eisenberg E: Systematic identification of edited microRNAs in the human brain. Genome Res 2012, 22(8):1533-1540.

50. Zhao D, McBride D, Nandi S, McQueen HA, McGrew MJ, Hocking PM, Lewis PD Sang HM, Clinton M: Somatic sex identity is cell autonomous in the chicken. Nature 2010, 464(7286):237-242.

51. Lewis BP, Burge CB, Bartel DP: Conserved seed pairing, often flanked by adenosines, indicates that thousands of human genes are microRNA targets. Cell 2005, 120(1):15-20

52. Morin RD, O'Connor MD, Griffith M, Kuchenbauer F, Delaney A, Prabhu AL, Zhao Y, McDonald H, Zeng T, Hirst M, et al: Application of massively parallel sequencing to microRNA profiling and discovery in human embryonic stem cells. Genome Res 2008, 18(4):610-621.

53. Marti E, Pantano L, Banez-Coronel M, Llorens F, Minones-Moyano E, Porta S, Sumoy L, Ferrer I, Estivill X: A myriad of miRNA variants in control and Huntington's disease brain regions detected by massively parallel sequencing. Nucleic Acids Res 2010, 38(20):7219-7235.

54. Berezikov E, Robine N, Samsonova A, Westholm JO, Naqvi A, Hung JH, Okamura K, Dai Q, Bortolamiol-Becet D, Martin R, et al: Deep annotation of drosophila melanogaster microRNAs yields insights into their processing, modification, and emergence. Genome Res 2011, 21(2):203-215.

55. Lee LW, Zhang S, Etheridge A, Ma L, Martin D, Galas D, Wang K: Complexity of the microRNA repertoire revealed by next-generation sequencing. RNA 2010, 16(11):2170-2180.

56. Fernandez-Valverde SL, Taft RJ, Mattick JS: Dynamic isomiR regulation in drosophila development. RNA 2010, 16(10):1881-1888.

57. Jones MR, Quinton LJ, Blahna MT, Neilson JR, Fu S, Ivanov AR, Wolf DA Mizgerd JP: Zcchc11-Dependent uridylation of microRNA directs cytokine expression. Nat Cell Biol 2009, 11(9):1157-1163.

58. Katoh T, Sakaguchi Y, Miyauchi K, Suzuki T, Kashiwabara S, Baba T: Selective stabilization of mammalian microRNAs by $3^{\prime}$ adenylation mediated by the cytoplasmic poly(a) polymerase GLD-2. Genes Dev 2009, 23(4):433-438.

59. Baccarini A, Chauhan H, Gardner TJ, Jayaprakash AD, Sachidanandam R, Brown BD: Kinetic analysis reveals the fate of a microRNA following target regulation in mammalian cells. Curr Biol, 21(5):369-376.

60. Birchler JA: Dosage compensation for the birds. Heredity (Edinb) 2009, 102(5):423-424

61. Baverstock PR, Adams M, Polkinghorne RW, Gelder M: A sex-linked enzyme in birds-Z-chromosome conservation but no dosage compensation. Nature 1982, 296(5859):763-766.

62. Kuroda Y, Arai N, Arita M, Teranishi M, Hori T, Harata M, Mizuno S: Absence of Z-chromosome inactivation for five genes in male chickens. Chromosome Res 2001, 9(6):457-468.

63. McQueen HA, McBride D, Miele G, Bird AP, Clinton M: Dosage compensation in birds. Curr Bio/ 2001, 11(4):253-257.

64. Ellegren $H$, Hultin-Rosenberg L, Brunstrom B, Dencker L, Kultima K, Scholz B: Faced with inequality: chicken do not have a general dosage compensation of sex-linked genes. BMC Biol 2007, 5:40.

65. Itoh Y, Melamed E, Yang X, Kampf K, Wang S, Yehya N, Van Nas A, Replogle K, Band MR, Clayton DF, et al: Dosage compensation is less effective in birds than in mammals. J Biol 2007, 6(1):2.

66. Itoh Y, Replogle K, Kim YH, Wade J, Clayton DF, Arnold AP: Sex bias and dosage compensation in the zebra finch versus chicken genomes: general and specialized patterns among birds. Genome Res 2010, 20(4):512-518.

67. Mank JE, Ellegren $\mathrm{H}$ : All dosage compensation is local: gene-by-gene regulation of sex-biased expression on the chicken $Z$ chromosome. Heredity (Edinb) 2009, 102(3):312-320.

68. Bisoni L, Batlle-Morera L, Bird AP, Suzuki M, McQueen HA: Female-specific hyperacetylation of histone $\mathrm{H} 4$ in the chicken $\mathrm{Z}$ chromosome. Chromosome Res 2005, 13(2):205-214. 
69. Melamed E, Arnold AP: Regional differences in dosage compensation on the chicken Z chromosome. Genome Biol 2007, 8(9):R202.

70. Reiner A, Laverghetta AV, Meade CA, Cuthbertson SL, Bottjer SW: An immunohistochemical and pathway tracing study of the striatopallidal organization of area $\mathrm{X}$ in the male zebra finch. J Comp Neurol 2004, 469(2):239-261.

71. Arnold AP: Sexual differentiation of the zebra finch song system: positive evidence, negative evidence, null hypotheses, and a paradigm shift. J Neurobiol 1997, 33(5):572-584

72. Tang YP, Peabody C, Tomaszycki ML, Wade J: Sexually dimorphic SCAMP1 expression in the forebrain motor pathway for song production of juvenile zebra finches. Dev Neurobiol 2007, 67(4):474-482.

73. Crooks GE, Hon G, Chandonia JM, Brenner SE: WebLogo: a sequence logo generator. Genome Res 2004, 14(6):1188-1190.

74. Livak KJ, Schmittgen TD: Analysis of relative gene expression data using real-time quantitative PCR and the 2(-delta delta $C(T)$ ) method. Methods 2001, 25(4):402-408.

doi:10.1186/1471-2164-13-727

Cite this article as: Luo et al: Genome-wide annotation and analysis of zebra finch microRNA repertoire reveal sex-biased expression. BMC

Genomics 2012 13:727.

\section{Submit your next manuscript to BioMed Central and take full advantage of:}

- Convenient online submission

- Thorough peer review

- No space constraints or color figure charges

- Immediate publication on acceptance

- Inclusion in PubMed, CAS, Scopus and Google Scholar

- Research which is freely available for redistribution 